\title{
BMJ Open Influence of occupation type on the association between sleep duration and impaired fasting glucose: results from a Chinese population-based study
}

Qingyun Lu, Shangxi Wu, Shiyu Wang, Jing Xiao

To cite: Lu Q, Wu S, Wang S, et al. Influence of occupation type on the association between sleep duration and impaired fasting glucose: results from a Chinese populationbased study. BMJ Open 2021;11:e042066. doi:10.1136/ bmjopen-2020-042066

- Prepublication history and additional supplemental materia for this paper are available online. To view these files, please visit the journal online (http://dx.doi.org/10.1136/ bmjopen-2020-042066).

Received 29 June 2020 Accepted 18 February 2021
Check for updates

(C) Author(s) (or their employer(s)) 2021. Re-use permitted under CC BY-NC. No commercial re-use. See rights and permissions. Published by BMJ.

Department of Epidemiology and Medical Statistics, Nantong University, Nantong, China

Correspondence to Dr Jing Xiao; jxiaont@163.com

\section{ABSTRACT}

Objectives Systematic evaluation of the influence of occupation type on the association between sleep-glucose metabolism

Design A cross-sectional study.

Setting The Nantong Metabolic Syndrome Study is a

Chinese population-based study.

Participants 20502 participants aged 18-74 years old. Intervention No intervention.

Primary and secondary outcome measures Impaired fasting glucose (IFG).

Results A total of 1503 participants (7.33\%) with a slightly longer sleep duration had IFG. After being stratified according to occupation, a sleep duration of $\geq 10$ hours daily corresponded to a 1.321 -fold risk of IFG (95\% Cl 1.071 to $1.628, p=0.0092)$ among moderate and heavy physical workers compared with those with a daily sleep duration of 7-9 hours. There was no significant relationship between sleep and IFG among other types of workers. Moreover, we discovered a gender difference in the influence of occupation on the sleep-IFG. A positive association among moderate and heavy physical men and a negative association among light or sedentary men were established, but not in unemployed men. However, a positive association was evident only in unemployed women; there was no significant association among other occupations. Conclusion This study highlights the role of occupation in the relationship of sleep-glucose metabolism. A gender difference was found to have been influenced by occupational types on the sleep-metabolic association.

\section{INTRODUCTION}

Sleep health is a global health concern. The National Sleep Foundation in the USA recommends that a healthy adult's sleep ranged 7-9 hours/day. According to the recent data from the Centers for Disease Control and Prevention, nearly $35.2 \%$ of the general US population had short sleep duration ( $<7$ hours daily). ${ }^{1}$ Cumulative evidence suggests that sleep duration, both less or excessive, greatly affects human health and metabolism, increasing the risk of various chronic diseases. ${ }^{2}$ Human evidence suggests that excessive sleep may increase the risk of metabolic disorders. ${ }^{3}$ Recent evidence suggested U-shaped relationships between sleep duration and both
Strengths and limitations of this study

- Based on a large-scale population-based crosssectional study.

- Highlights the role of occupation in the sleep-glucose metabolism relationship.

- Self-reported information on sleep duration and physical activity data, the cross-sectional nature of data collection and residual confounders might exist, which may introduce bias.

pre-diabetes and diabetes, ${ }^{45}$ indicating that both less and excessive sleep durations are associated with a high risk of diabetes.

Previous evidence has shown that sociodemographic factors, ${ }^{6}$ psychosocial stress ${ }^{7}$ and lifestyle ${ }^{8}$ significantly influence sleep quality and duration. Furthermore, occupation is an important indicator of an individual's social and life status, summarising the social position, income, educational level, job stress, workload and number of work hours, especially physical activities performed at work. ${ }^{9}$ An observational study in the USA revealed that manufacturers had shorter sleep duration than other workers. ${ }^{10}$ Similarly, the Chinese Suboptimal Health Study in 2008 also discovered that farmers had a longer sleep duration than non-farmers. ${ }^{11}$ In addition, previous evidence indicated that all aforementioned occupations and related factors were linked with metabolic disorders. Impaired fasting glucose (IFG) is considered an early metabolic abnormality preceding diabetes. It is reported that patients with IFG were four times likely to have diabetes after 1 year compared with normoglycaemic people. ${ }^{12}$ Therefore, occupation is related to both sleep and metabolism; thus, it is reasonable to suspect that occupation might have a critical influence on the sleep-IFG association. However, only few epidemiological studies comprehensively examined 
the sleep-glucose metabolism association; most studies regarded occupation as only a confounding variable. ${ }^{13-15}$ In this study, we aimed to comprehensively evaluate the influence of occupation types on the sleep-IFG association. The regional factor is an important factor that might largely influence not only the sleep patterns but also the occupational types ${ }^{16}$; this discrepancy is significant especially in China. Nevertheless, there are currently less data from rural areas. Thus, in this study, we used the data from a large-scale survey on two rural towns in Nantong, including a relatively high proportion of agricultural and heavy manual workers.

\section{METHODS}

Patient and public involvement

The Nantong Metabolic Syndrome Study is a cross-sectional study conducted in Nantong City, Jiangxi Province of China, which recruited a total of 20502 participants (6997 men and 13505 women) of 24519 residents aged 18-74 years from two rural towns in Nantong from July 2007 to August 2008. The response rate was $83.6 \%$, and the participant selection criteria are illustrated in figure 1 . The detailed proposal of this study has been published. ${ }^{15} 17$

Trained researchers conducted face-to-face interviews in accordance with standard protocols to collect information on sociodemographic characteristics, occupational types, personal medical history, family history of chronic diseases, history of medication use, current smoking status, alcohol consumption, occupational physical activity (OPA), leisure physical activity (LPA) and sleep duration. Moreover, height, weight and waist circumference in centimetre were also measured following a standard protocol. Body Mass Index (BMI) was calculated as weight in kilogram divided by height (in metre) squared.

Total 24,519 rural residents recruited from two townships

Refusal (787)

23,732 residents

being out of the area $(1,768)$

\section{1,964 residents}

poor health or hearing

problems $(1,462)$

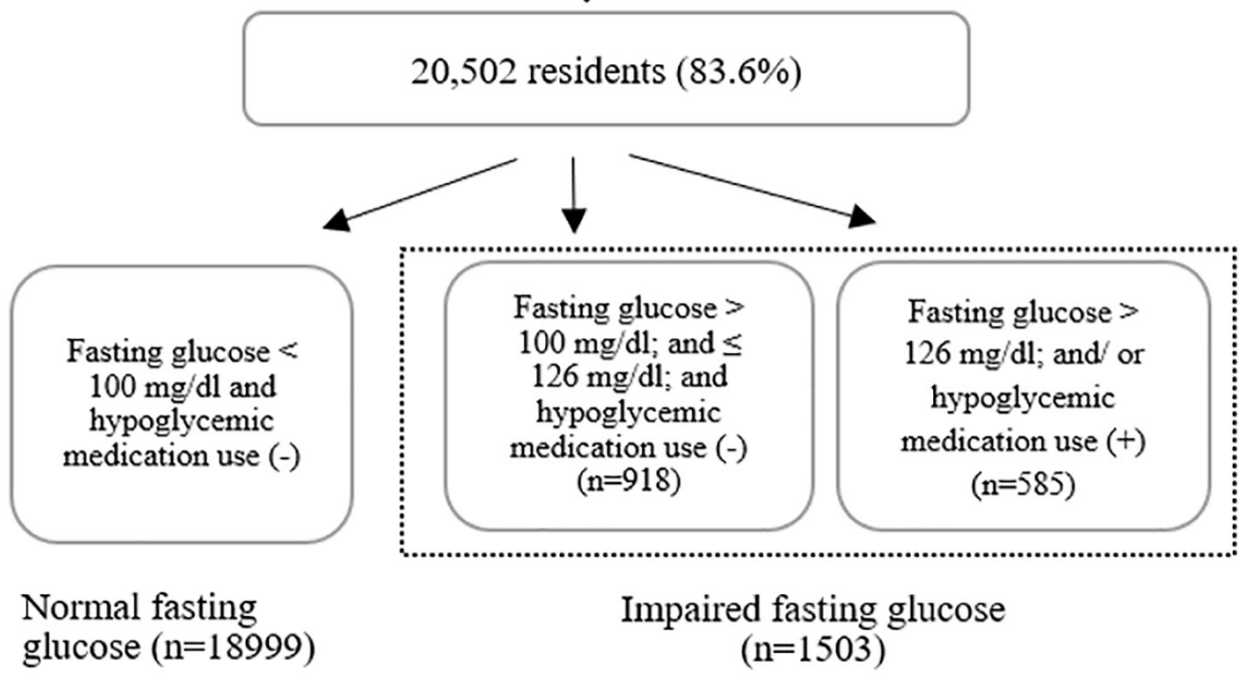

Figure 1 Participant selection. 
Venous blood samples were obtained from each participant after fasting for $\geq 10$ hours, which were then shipped within 24 hours to the central laboratory and stored frozen at $-80^{\circ}$ until further analysis. Plasma fasting glucose concentrations were determined using standardised enzymatic methods. We identified the IFG according to fasting plasma glucose levels, with fasting glucose levels between 100 and $126 \mathrm{mg} / \mathrm{dL}$ and without the use of hypoglycaemic medications in the past 2 weeks. ${ }^{18}$ If the participants reported having been diagnosed with diabetes or having taken antidiabetes drugs, they were identified as diabetes.

The hours of sleep duration were determined by the following questions: 'at what time do you go to bed at night during the weekday?' and 'at what time do you wake up during the weekday?' The sleep duration during the weekends were also queried and recorded. Daily sleeping hours were calculated as an overall average sleep duration (sleep duration in weekday $\times 5+$ sleep duration in weekends $\times 2$ ) /7). We categorised the participants into three groups according to their daily sleep duration: $<7,7-\leq 9$ and $>9$ hours. ${ }^{5}$ Considering the large discrepancy in occupation between China and the USA, we did not use International Standard Classification of occupations (ISCO) for classification of occupation. We used the China National Standard Physical Labour Intensity Grade (GB 3869-83) to construct the occupational categories, which has the widely used and accepted occupational categories in China. A total of 18 standard Chinese occupational types were included: farmer, transport worker, construction worker, steelworker, driver, electrician, bench worker, metal worker, carpenter, retail sales worker, service worker, installation worker, teacher, civil servant, specialist, clerk and technician. One primary occupation was reported for each participant. These occupations were clustered into three types based on their intensity of physical activity at work: medium and heavy physical workers (transport worker, construction worker, steel worker and farmer), light physical workers (driver, electrician, bench worker, metal worker, carpenter, installation worker, retail sales worker and service worker) and sedentary workers (civil servant, teacher, clerk, technician and computer repairman). Only $6.4 \%$ of participants were retired, unemployed or homemakers; we pooled these participants with unemployed participants. ${ }^{11}{ }^{19}$ In addition, we calculated the motion equivalent of OPA level for each participant. The data on intensity and duration (average daily hours) of OPA were obtained through a standardised questionnaire. OPA was classified as sedentary, light, moderate and heavy. Moreover, daily metabolic equivalents (METs) of OPA were calculated based on the hours of physical activity and energy requirements during work: light intensity, 3.3 METs (based on walking METs); moderate intensity, 4.0 METs; and vigorous intensity, 8.0 METs. ${ }^{20}$ Sedentary behaviour refers to activities that involve energy expenditure at the level of 1.0-1.5 METs.

Information on potential confounders, including age at the time of the interview, education levels (primary school or under, middle school, and high school or above), monthly personal income $(\leq ¥ 500 \quad(\$ 75), 501 \quad(\$ 76)-$ $¥ 1000(\$ 150)$, and $\geq ¥ 1001$ (\$151)), current smoking status (yes or no), current alcohol consumption (yes or no) and physical activity (vigorous, moderate, light and without LPA) were gathered. Current smokers were classified as those who smoked at least 100 cigarettes in their lifetime. Current alcohol drinkers were defined as participants who had beverage consumption containing approximately $0.5 \mathrm{oz}$ of pure alcohol in 1 month. ${ }^{21}$ Furthermore, information on LPA was obtained using a standardised questionnaire and classified into four categories: none, light (qi gong and some stretching exercises), moderate (jogging and dancing) and vigorous (basketball and badminton). ${ }^{22}$ Similar to OPA, daily METs of LPA were calculated accordingly.

We calculated the means \pm SD for continuous variables and frequency and percentage for categorical variables. Furthermore, we calculated the case number and the prevalence of IFG. t-Tests or Kruskal-Wallis tests were performed to compare the differences in continuous variables between IFG and normal participants, wherever appropriate. The $\chi^{2}$ tests were used for categorical variables. Crude and multivariable logistic regression models were used to estimate the ORs and their 95\% CIs, and to assess the sleep-IFG associations in different occupational types following the adjustment for potential confounders, including age at interview, marital status, education levels, personal income, current smoking status, current alcohol consumption, BMI and LPA. We also conducted crude and multivariable logistic regression models to calculate the ORs and to assess the association of sleep duration and occupational types with IFG risk. Separately, we further evaluated the influence of occupational types on the sleep-IFG association in men and women. In the linear regression, both crude and adjusted models were used to estimate the ORs of IFG risk for each 1-hour increase in sleep duration and each 1-MET-hour/week increase in physical activity.

All statistical analyses were performed using SAS statistical software V.9.4. A two-tailed $p$ value of $<0.05$ was considered statistically significant.

\section{RESULTS}

In total, 20502 participants aged $54 \pm 12$ years were included herein; among them, $34.13 \%$ were men. A total of 1503 participants $(7.33 \%)$ were diagnosed with IFG. Participants with IFG tended to be older, had a higher $\mathrm{BMI}$ and wider waist circumference, and had lower education levels and personal income ( $\mathrm{p}<0.05$ for all). Moreover, participants with IFG were likely to have jobs with lower physical activity and less sleep duration compared with participants with normal fasting glucose $(p<0.05$ for all) (table 1).

After adjusting for age at the interview, sex, BMI, marital status, education levels, personal income per month, 


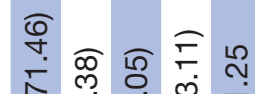

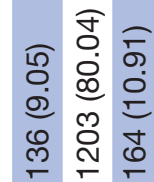
吾

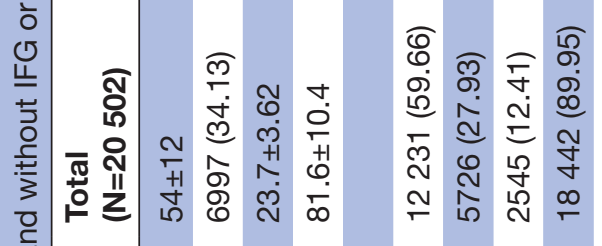

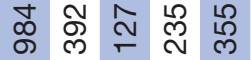

ᄃ

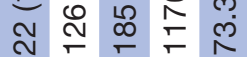

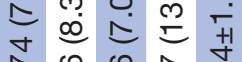

走

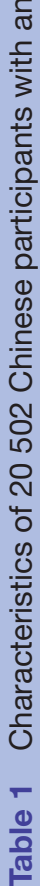

गิ

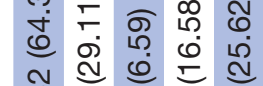

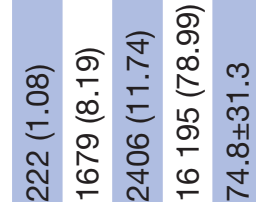

蛋

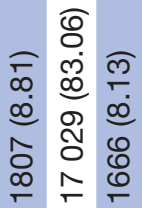

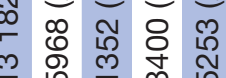

a)

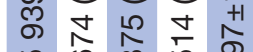

吕

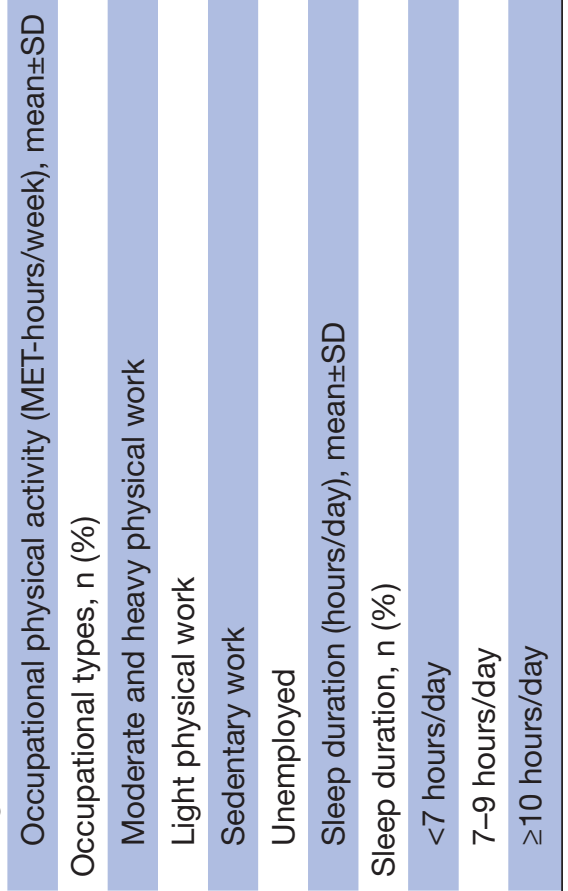

\section{产}

○

की

㐨

$\frac{\grave{m}}{0}$

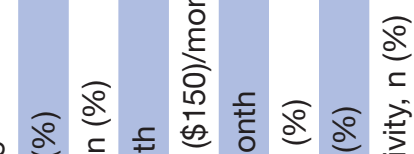

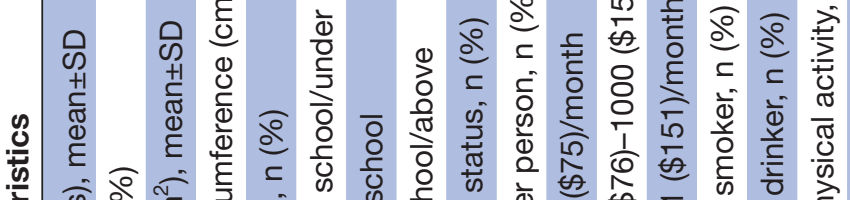

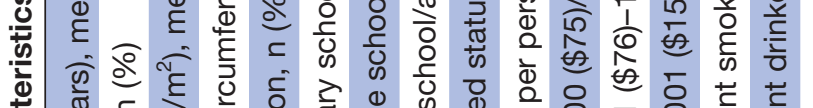

$-$

$\frac{\frac{0}{0}}{\frac{0}{๘}}$ 
current smoking and drinking status and LPA, longer sleep duration ( $\geq 10$ hours daily) significantly corresponded to a high risk of IFG (OR 1321, 95\% CI 1.106 to $1.579, \mathrm{p}=0.0022$ ). Meanwhile, OPA (OR $0.996,95 \% \mathrm{CI}$ 0.994 to $0.998, \mathrm{p}=0.0001$ ) or moderate and heavy physical work (OR 0.696, 95\% CI 0.554 to $0.875, \mathrm{p}=0.0019$ ) had a negative correlation with the risk of IFG (table 2).

After stratification by occupational types, as the amount of OPA decreased, the prevalence of IFG was increased from $6.74 \%$ among moderate and heavy physical workers to $13.01 \%$ among unemployed workers, and there was a positive association between sleep duration and IFG among moderate and heavy physical workers, and every 1-hour increase in daily sleep duration equates to $6.6 \%$ (OR $1.066,95 \%$ CI 1.005 to $1.130, \mathrm{p}=0.0344$ ) increase in the risk of IFG. Nonetheless, longer sleep duration was concomitant to a higher risk of IFG among moderate and heavy physical workers (OR 1.321, 95\% CI 1.071 to 1.628, $\mathrm{p}=0.0092$ ) compared with workers with 7-9 hours of daily sleep duration. However, in other worker groups, light physical workers, sedentary workers and unemployed workers, there were non-significant sleep-IFG associations (all $\mathrm{p}$ values of $>0.05$ ) (table 3 ). We further evaluated the influence of occupational types on the association between sleep duration and risk of IFG after excluding patients with diabetes and found consistent results (online supplemental table).

We further evaluated the influence of occupational types on the sleep-IFG association between men and women (table 4). In men, sleep duration in different occupational types had a different influence on the risk of IFG. For moderate and heavy physical workers, longer duration of sleep indicated a higher risk of IFG; every 1-hour increase in daily sleep duration corresponded to a 1.127 times higher risk of IFG (95\% CI 1.015 to 1.251 , $\mathrm{p}=0.0253$ ). In contrast to workers with a daily sleep duration of 7-9 hours, those with $\geq 10$ hours of sleep duration per day likely had a high risk of IFG (OR $1.734,95 \%$ CI 1.191 to $2.524, \mathrm{p}=0.0041$ ).

However, the associations were the reverse among light or sedentary workers; longer daily sleep duration was associated with a lower risk of IFG. Every 1-hour decrease in daily sleep duration corresponded to a $22.9 \%$ lower risk of IFG (95\% CI 0.601 to 0.989 , $\mathrm{p}=0.0408$ ) among light physical workers and a $27.5 \%$ lower risk of IFG (95\% CI 0.559 to $0.941, \mathrm{p}=0.0156$ ) among sedentary physical workers. In comparison to those with 7-9 hours of daily sleep, workers with a sleep duration $<7$ hours tended to have a 2.136 times risk (95\% CI 1.035 to $4.409, \mathrm{p}=0.0401$ ) among light physical workers and 2.134 times risk (95\% CI 0.559 to 0.941 , $\mathrm{p}=0.0428$ ) among sedentary physical workers, respectively. Non-significant associations were found between unemployed men, while among women, only a positive sleep-IFG association among unemployed women (OR $1.236,95 \%$ CI 1.055 to $1.449, \mathrm{p}=0.0088$ ) was determined. However, there was no significant association among other types of occupation. 

cial for their glucose metabolic health. For unemployed women, a shorter sleep duration indicated a low risk of IFG.

The influence of occupational types on the sleepIFG association might be partially caused by the gender difference in occupational types. We found that heavy and moderate physical workers had the lowest personal income, education level and LPA; however, this group had the longest sleep duration in both genders. Men in this group had the longest sleep duration and increased IFG risk. In general, it was believed that those engaged in more physical activity may sleep more; however, Martins et al reported that physical exertion at work may not improve sleep quality. ${ }^{23}$ Unlike LPA, OPA is characterised by constrained activities related to specific tasks with less rest. ${ }^{24}$ Studies have highlighted that LPA enhanced sleep quality and duration, ${ }^{25} 26$ while highly intense OPA, and even those with heavy physical work, had adverse effects on sleep. ${ }^{2728}$ A longer sleep duration among heavy physical workers might be compensated for the physical exertion at work. This might partially explain the longer sleep duration related with high risk of IFG among heavy physical workers. Moreover, low socioeconomic status accompanied by complications of immobility ${ }^{29}$ were strongly associated with long sleep duration. Therefore, heavy-OPA men with low socioeconomic status and long sleep duration were at a high risk of IFG. However, there was a non-significant association between long sleep duration and increased IFG in women who performed heavy physical work. Since most of the women in this group were farmers, whereas the occupational types of men were those of transport worker, build worker and steelworker, men had higher levels of OPA with considerable productivity and less rest than women.

Our findings demonstrated that short sleep duration was significantly associated with increased risk of IFG only in men of light or sedentary physical work. The effect of short sleep duration on IFG may be exacerbated by low OPA level. Men with light or sedentary physical work generally had high income or education levels and OPA levels but with more screening time and sedentary time. A study only supported the synergic effect between low OPA and short sleep duration on insulin resistance among non-diabetic Chinese adults; conversely, this study included both genders. ${ }^{30}$ Consistently, a study from Sweden confirmed that short sleep duration in men was associated with an increased incidence of diabetes ${ }^{31}$ and 


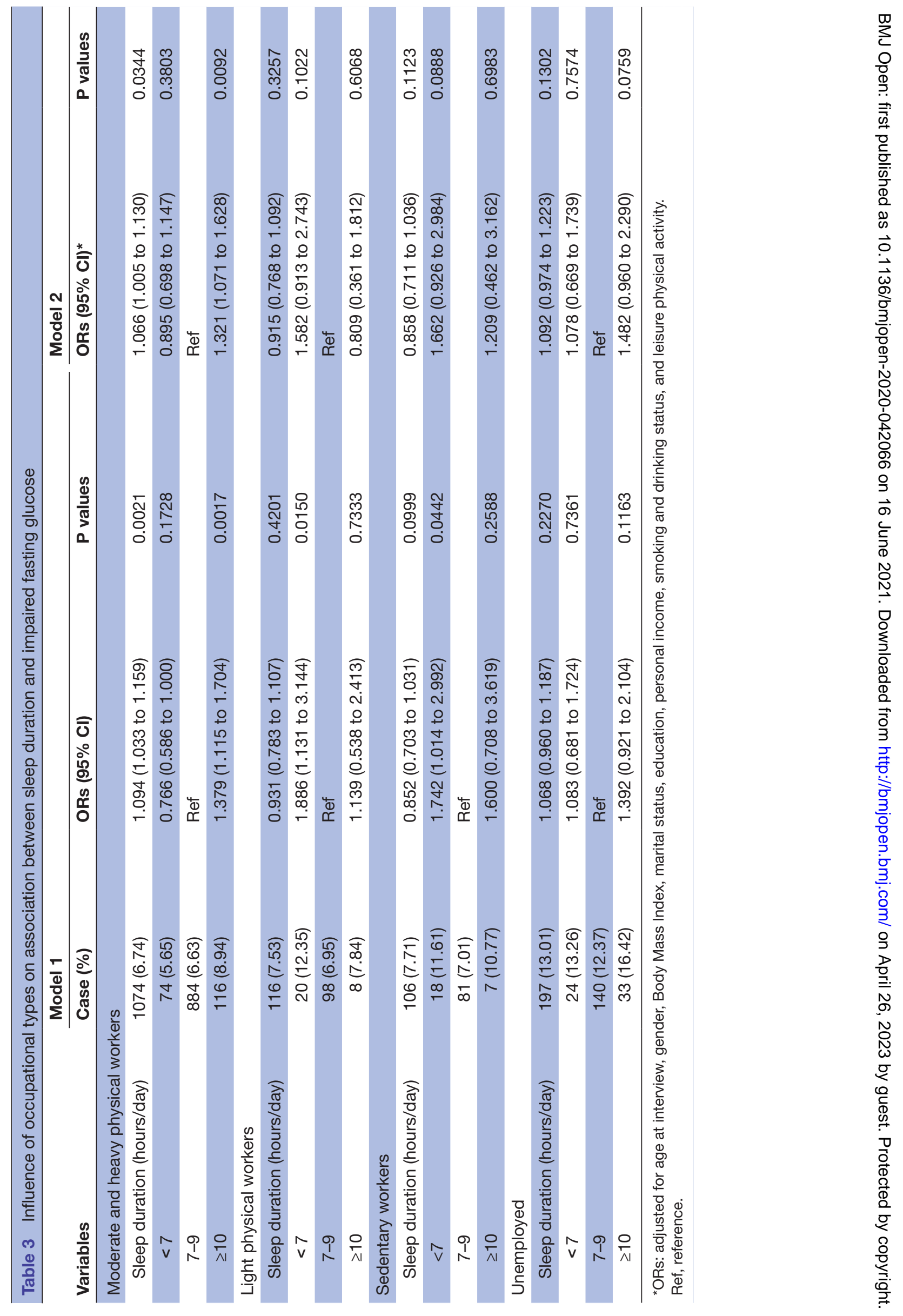




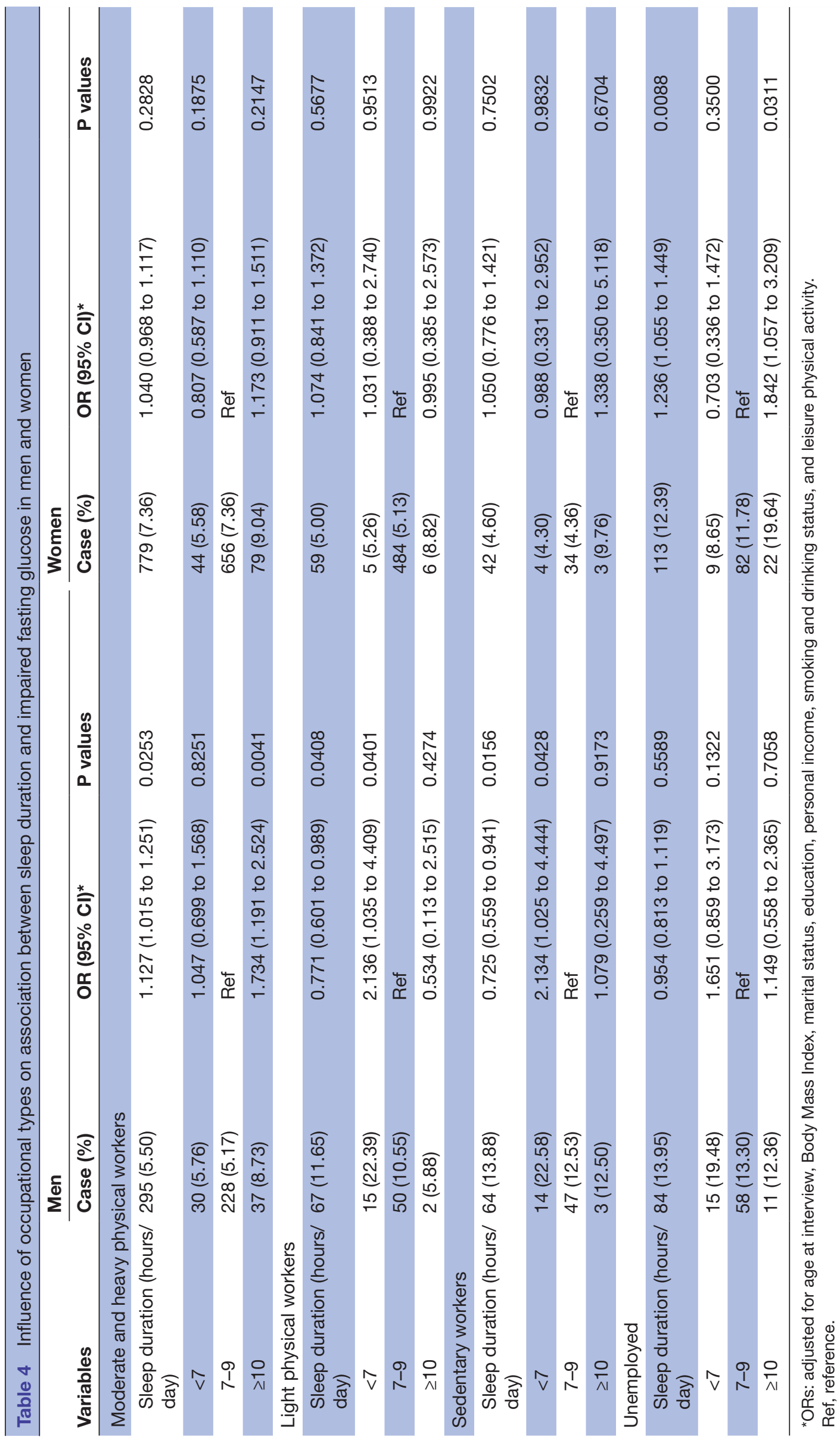

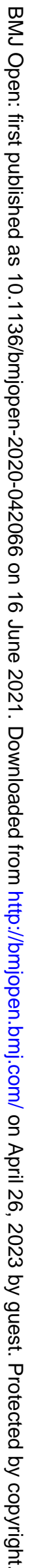


impaired glucose tolerance. ${ }^{32}$ Recent studies reported that both short and long sleep durations were associated with diabetes in men but not in women. ${ }^{3-35}$

Regarding women, some studies have suggested a significant U-shaped association between sleep duration and diabetes, especially among middle-aged women. ${ }^{36} 37$ Our results demonstrated that long sleep duration did not increase the risk of IFG in heavy physical, light physical or sedentary workers but increased the risk of IFG in unemployed women. These divergent findings may be attributed to differences in age, sample size and fasting glucose level definitions. Some studies reported a strong association between habitual long sleepers and diabetes among middle-aged women. ${ }^{36}{ }^{37}$ Unemployed women had a relatively longer sleep duration than employed women with no or low OPA level and few domestic chores and had significantly higher IFG risk. Furthermore, lower levels of total physical activity in unemployed women might exacerbate sleep quality. ${ }^{38}$

Our study has several limitations. First, sleep duration and physical activity data were obtained from self-reports. Second, the cross-sectional nature of data collection means that we were unable to draw causal inferences. Third, there might be a healthy worker effect, which cannot be excluded. Fourth, the regional factor might have influenced both OPA and sleep patterns. This study was focused on the population from rural areas, which is difficult to extend to urban areas, especially for those metropolitan. Another limitation is that most of the population of our study population was $>50$ years old, and only a few people reported having a shift work, which might limit our conclusions. Moreover, other factors, such as stress and menopausal status, can interact with sleep, which were not included in this study. Finally, we chose to combine IFG and diabetes mellitus and relied on a single plasma fasting glucose measurement, which could introduce a misclassification bias.

\section{CONCLUSION}

The main findings of this study comprise the influence of occupational gender difference on the relationship between sleep duration and IFG risk, which suggested that sleep duration should be decided on the basis of occupation types. Under a large population-based survey, we discovered that occupational type may influence sleep and glucose metabolism among men but not in women. Moreover, the direction of the sleep-glucose metabolic relationship is dependent on physical occupational types.

Contributors All authors conceived the study. QYL and SWu contributed to the initial draft and review. Their contributions to the article are equal. QYL and SWu contributed to the conception, design and review. QYL and JX contributed to the statistical analysis, data interpretation and review. All authors approved the final version of the manuscript.

Funding This study was supported by the National Natural Science Foundation of China (grant number 81502827).

Competing interests None declared.
Patient consent for publication Not required.

Ethics approval The study protocol was approved by the medical ethical committee of Nantong University (number 202056).

Provenance and peer review Not commissioned; externally peer reviewed.

Data availability statement Data are available upon reasonable request. The data that support the findings of this study are available from the corresponding author upon reasonable request.

Supplemental material This content has been supplied by the author(s). It has not been vetted by BMJ Publishing Group Limited (BMJ) and may not have been peer-reviewed. Any opinions or recommendations discussed are solely those of the author(s) and are not endorsed by BMJ. BMJ disclaims all liability and responsibility arising from any reliance placed on the content. Where the content includes any translated material, BMJ does not warrant the accuracy and reliability of the translations (including but not limited to local regulations, clinical guidelines, terminology, drug names and drug dosages), and is not responsible for any error and/or omissions arising from translation and adaptation or otherwise.

Open access This is an open access article distributed in accordance with the Creative Commons Attribution Non Commercial (CC BY-NC 4.0) license, which permits others to distribute, remix, adapt, build upon this work non-commercially, and license their derivative works on different terms, provided the original work is properly cited, appropriate credit is given, any changes made indicated, and the use is non-commercial. See: http://creativecommons.org/licenses/by-nc/4.0/.

\section{ORCID iD}

Jing Xiao http://orcid.org/0000-0003-1462-2065

\section{REFERENCES}

1 Zheng X, Zhang W, Hu X. Different concentrations of lipopolysaccharide regulate barrier function through the PI3K/Akt signalling pathway in human pulmonary microvascular endothelial cells. Sci Rep 2018;8:9963.

$2 \mathrm{Xu} \mathrm{Y}$, Wang L, He J, et al. Prevalence and control of diabetes in Chinese adults. JAMA 2013;310:948-59.

3 Bliwise DL, Young TB. The parable of parabola: what the U-shaped curve can and cannot tell us about sleep. Sleep 2007;30:1614-5.

4 Bauer M, Glenn T, Whybrow PC, et al. Changes in self-reported sleep duration predict mood changes in bipolar disorder. Psychol Med 2008;38:1069-71.

5 Shan Z, Ma H, Xie M, et al. Sleep duration and risk of type 2 diabetes: a meta-analysis of prospective studies. Diabetes Care 2015;38:529-37.

6 Gamaldo AA, Gamaldo CE, Allaire JC, et al. Sleep complaints in older blacks: do demographic and health indices explain poor sleep quality and duration? J Clin Sleep Med 2014;10:725-31.

7 Tom SE, Berenson AB. Associations between poor sleep quality and psychosocial stress with obesity in reproductive-age women of lower socioeconomic status. Womens Health Issues 2013;23:e295-300.

8 Hoefelmann LP, Lopes AdaS, Silva KSda, et al. Lifestyle, selfreported morbidities, and poor sleep quality among Brazilian workers. Sleep Med 2012;13:1198-201.

9 Akerstedt T, Fredlund P, Gillberg M, et al. Work load and work hours in relation to disturbed sleep and fatigue in a large representative sample. J Psychosom Res 2002;53:585-8.

10 Luan Z-G, Naranpurev M, Ma X-C. Treatment of low molecular weight heparin inhibits systemic inflammation and prevents endotoxininduced acute lung injury in rats. Inflammation 2014;37:924-32.

11 Sun W, Yu Y, Yuan J, et al. Sleep duration and quality among different occupations--China national study. PLoS One 2015;10:e0117700.

12 Gerstein HC, Santaguida P, Raina P, et al. Annual incidence and relative risk of diabetes in people with various categories of dysglycemia: a systematic overview and meta-analysis of prospective studies. Diabetes Res Clin Pract 2007;78:305-12.

13 Tentolouris N, Andrianakos A, Karanikolas G, et al. Type 2 diabetes mellitus is associated with obesity, smoking and low socioeconomic status in large and representative samples of rural, urban, and suburban adult Greek populations. Hormones 2012;11:458-67.

14 Jackson CL, Redline S, Kawachi I, et al. Association between sleep duration and diabetes in black and white adults. Diabetes Care 2013;36:3557-65.

15 Lou P, Chen P, Zhang L, et al. Interaction of sleep quality and sleep duration on impaired fasting glucose: a population-based crosssectional survey in China. BMJ Open 2014;4:e004436. 
16 Beijamini F, Knutson KL, Lorenzi-Filho G, et al. Timing and quality of sleep in a rural Brazilian family-based cohort, the Baependi heart study. Sci Rep 2016;6:39283.

17 Xiao J, Wu C, Xu G, et al. Association of physical activity with risk of metabolic syndrome: findings from a cross-sectional study conducted in rural area, Nantong, China. J Sports Sci 2016;34:1839-48.

18 Bakker JP, Weng J, Wang R, et al. Associations between obstructive sleep apnea, sleep duration, and abnormal fasting glucose. The multi-ethnic study of atherosclerosis. Am J Respir Crit Care Med 2015;192:745-53.

19 Du H, Bennett D, Li L, et al. Physical activity and sedentary leisure time and their associations with BMI, waist circumference, and percentage body fat in 0.5 million adults: the China Kadoorie Biobank study. Am J Clin Nutr 2013;97:487-96.

20 Craig CL, Marshall AL, Sjöström M, et al. International physical activity questionnaire: 12 -country reliability and validity. Med Sci Sports Exerc 2003;35:1381-95.

21 Shu XO, Hatch MC, Mills J, et al. Maternal smoking, alcohol drinking, caffeine consumption, and fetal growth: results from a prospective study. Epidemiology 1995;6:115-20.

22 IRAQ group. Guideline for data procssing and analysis of the International physical activity questionnaire (IPAQ) short form, 2020. Available: https://sites.google.com/site/theipaq/scoring-protocol [Accessed 18 Jan 2010].

23 Martins AJ, Vasconcelos SP, Skene DJ, et al. Effects of physical activity at work and life-style on sleep in workers from an Amazonian Extractivist reserve. Sleep Sci 2016;9:289-94.

24 Holtermann A. Occupational and leisure-time physical activity and coronary heart disease. Occup Environ Med 2015;72:615-6.

25 de Castro Toledo Guimaraes LH, de Carvalho LBC, Yanaguibashi $\mathrm{G}$, et al. Physically active elderly women sleep more and better than sedentary women. Sleep Med 2008;9:488-93.

26 Reid KJ, Baron KG, Lu B, et al. Aerobic exercise improves selfreported sleep and quality of life in older adults with insomnia. Sleep Med 2010;11:934-40.

27 Geroldi C, Frisoni GB, Rozzini R, et al. Principal lifetime occupation and sleep quality in the elderly. Gerontology 1996;42:163-9.
28 Spiegel K, Tasali E, Penev P, et al. Brief communication: sleep curtailment in healthy young men is associated with decreased leptin levels, elevated ghrelin levels, and increased hunger and appetite. Ann Intern Med 2004;141:846-50.

29 Patel SR, Malhotra A, Gottlieb DJ, et al. Correlates of long sleep duration. Sleep 2006;29:881-9.

30 Zuo H, Shi Z, Yuan B, et al. Interaction between physical activity and sleep duration in relation to insulin resistance among non-diabetic Chinese adults. BMC Public Health 2012;12:247.

31 Mallon L, Broman J-E, Hetta J. High incidence of diabetes in men with sleep complaints or short sleep duration: a 12-year follow-up study of a middle-aged population. Diabetes Care 2005;28:2762-7.

32 Andersson S, Ekman I, Friberg F, et al. The association between self-reported lack of sleep, low vitality and impaired glucose tolerance: a Swedish cross-sectional study. BMC Public Health 2013;13:700

33 Cumberbatch CG, Younger NO, Ferguson TS, et al. Reported hours of sleep, diabetes prevalence and glucose control in Jamaican adults: analysis from the Jamaica lifestyle survey 2007-2008. Int J Endocrinol 2011;2011:1-8.

34 Bai Y, Li X, Wang K, et al. Association of shift-work, daytime napping, and nighttime sleep with cancer incidence and cancer-caused mortality in Dongfeng-tongji cohort study. Ann Med 2016;48:641-51.

35 Rutters F, Besson $\mathrm{H}$, Walker M, et al. The association between sleep duration, insulin sensitivity, and $\beta$-cell function: the EGIR-RISC study. $J$ Clin Endocrinol Metab 2016;101:3272-80.

36 Tuomilehto $\mathrm{H}$, Peltonen $\mathrm{M}$, Partinen $\mathrm{M}$, et al. Sleep duration is associated with an increased risk for the prevalence of type 2 diabetes in middle-aged women - The FIN-D2D survey. Sleep Med 2008;9:221-7.

37 Shadyab AH, Kritz-Silverstein D, Laughlin GA, et al. EthnicSpecific associations of sleep duration and daytime napping with prevalent type 2 diabetes in postmenopausal women. Sleep Med 2015;16:243-9.

38 Niţiă O, Graur LI, Popescu DS, et al. Socio-Demographic and lifestyle characteristics associated with the risk for obstructive sleep apnea syndrome in a rural population. Rev Med Chir Soc Med Nat lasi 2012;116:773-9. 